\title{
Note on Uniqueness of Canonical Commutation Relations
}

R. Arnowitt and S. Deser

Citation: 4, (1963); doi: 10.1063/1.1703998

View online: http://dx.doi.org/10.1063/1.1703998

View Table of Contents: http://aip.scitation.org/toc/jmp/4/5

Published by the American Institute of Physics 


\title{
Note on Uniqueness of Canonical Commutation Relations*
}

\author{
R. Arnowitt and S. Deser \\ Department of Physics, Brandeis University, Waltham, Massachusetts
}

(Received 28 May 1962)

\begin{abstract}
It has been pointed out by Wigner that the consistency requirement between the Lagrange and Heisenberg equations of motion does not uniquely determine the canonical commutation relations, at least for one-dimensional systems. It is shown here that this ambiguity does not arise in local field theory whose basic equal-time commutators commute with the translation operator.
\end{abstract}

\section{INTRODUCTION}

$\mathrm{I}^{\mathrm{N}}$ $N$ the course of investigating ${ }^{1}$ the nature of the axioms underlying the Schwinger Action Principle, $^{2}$ it was found that the equal time (anti) commutator between a field and its conjugate momentum, $\left[\phi(\mathbf{r}), \pi\left(\mathbf{r}^{\prime}\right)\right]_{\star}$, could only be reduced to the form

$$
\left[\phi(\mathbf{r}), \pi\left(\mathbf{r}^{\prime}\right)\right]_{-}=i \delta^{3}\left(\mathbf{r}-\mathbf{r}^{\prime}\right)[1+F],
$$

where $F$ is not necessarily a $c$ number. This reduction was performed on the basis of quite general assumptions, as described in reference 1 .

In attempting to see whether $F$ could be forced to zero purely on consistency grounds (rather than by an additional postulate), the freedom of performing arbitrary unitary transformations in the theory was employed. Thus, consider the change generated by the infinitesimal operator $U \simeq 1+$ $i G\left(G^{+}=G\right), G=\int d^{3} r\left[f_{1}(\phi, \pi) \delta \phi+f_{2}(\phi, \pi) \delta \pi\right]$. The fundamental commutation relations then enable one to evaluate the change $\Delta R$ of any function $R(\phi, \pi)$, according to the definition $\Delta R \equiv-i[G, R]$. In general, $\Delta R$ will involve the operator $F$. For the special case in which $R$ is taken to be the Hamiltonian $H$ of the system, this change may be explicitly evaluated in another way using the Heisenberg equations of motion. Thus, in

$$
\Delta H=i[H, G]=d G / d t,
$$

the time derivative $d G\rangle d t$ depends on $\dot{\phi}$ and $\dot{\pi}$. The latter derivatives may be evaluated by using the (by assumption) identical Lagrange equations in which $F$ does not enter. The consistency of the two evaluations provides a stringent requirement on $F$. Indeed, if $F$ is to be a $c$ number, the above condition requires it to vanish (as will be shown below); however, q-number $F$ 's could not be so

\footnotetext{
* Supported in part by the Air Force Office of Scientific Research and the National Science Foundation.

1 R. Arnowitt and S. Deser, J. Math. Phys., 3, 637 (1962)

2 J. Schwinger, Phys. Rev. 82, 914 (1951); and 91, 713 (1953).
}

eliminated. An example of this situation had, in fact, been given some time ago by Wigner ${ }^{3}$ for the one-dimensional harmonic oscillator. Here, consistency between commutation relations and equations of motion still allowed $F$ to be any one of a one-parameter class of operators,

$$
F=\left(2 E_{0} / \hbar \omega-1\right) \exp \left[i \pi\left(H-E_{0}\right) / \hbar \omega\right],
$$

$\left(E_{0}\right.$ arbitrary $\left.{ }^{4}\right)$ anticommuting with both $p$ and $q$. However, we show here that, for local field theory whose basic equal-time commutators commute with the translation operator, $F$ is necessarily zero in order that the Lagrange and Heisenberg equations agree.

\section{DERIVATION}

Consider the general field theoretical system in first-order form. ${ }^{1}$ For convenience, the field variables (which may be taken to be Hermitian) may be arranged in a column symbol array with entries $\chi_{a}(x),(a=1 \cdots N)$. In the first-order description, the $\chi_{a}$ include both the field amplitudes and their conjugate momenta. Thus, the equal-time commutator (or anticommutator) $\left[\chi_{a}(\mathbf{r}), \chi_{b}\left(\mathbf{r}^{\prime}\right)\right]_{\text {. in- }}$ cludes all the usual commutation (or anticommutation) relations. In general, however, not all field variables are independent, some being functions of the rest if some of the Lagrange equations are constraint equations. The independent variables in the general case will be denoted by $\phi_{a}, a=1 \cdots M$, $M \leq N$. The commutators $\left[\phi_{a}(\mathbf{r}), \phi_{b}\left(\mathbf{r}^{\prime}\right)\right]_{*}$ are then a complete set of relations; any commutators involving the dependent variables are determined by them. In general, one may write

$$
\left[\boldsymbol{\phi}_{a}(\mathbf{r}), \phi_{b}\left(\mathbf{r}^{\prime}\right)\right]_{\star}=i f_{a b} \delta^{3}\left(\mathbf{r}-\mathbf{r}^{\prime}\right),
$$

${ }^{3}$ E. P. Wigner, Phys. Rev. 77, 711 (1950).

4 Note here $E_{0}$ represents the ground-state energy, which need not be $\frac{1}{2} \hbar \omega$, with commutation relations of the type of Eq. (1). However, the eigenspectrum of $H-E^{0}$ is still $n \hbar \omega$ and so $F$ is correctly Hermitian. 
where $f_{a b}$ may be an operator. The term $\delta^{3}\left(\mathbf{r}-\mathbf{r}^{\prime}\right)$ is a consequence of the locality assumption. ${ }^{5}$ It is also assumed that $f_{a b}$ is independent of the center of mass coordinate $\mathbf{r}+\mathbf{r}^{\prime}$. This assumption of translational invariance ${ }^{6}$ is equivalent to requiring that $f_{a b}$ and the translation generator of the theory $U$ commute, so that the commutators at all space points are numerically equal. This form of the translational invariance requirement is stronger than one in which the commutators are just required to be form invariant under translation. (The latter condition could be satisfied by an operator $f_{a b}$, depending on $\mathbf{r}+\mathbf{r}^{\prime}$ and translated by $U$.) For (anti) commutators, the operators $f_{a b}$ must be (skew-) Hermitian in the Hilbert space, and symmetric (antisymmetric) in the matrix indices $a b$. To prove that $f_{a b}$ is, in fact, a $c$-number matrix, consider the double "commutator"

$$
K \equiv\left[\phi_{a}(\mathbf{r}),\left[\phi_{b}\left(\mathbf{r}^{\prime}\right), \phi_{c}\left(\mathbf{r}^{\prime \prime}\right)\right]_{ \pm}\right],
$$

where the \pm signs refer to the Fermi (Bose) cases. The quantity $K$ vanishes for $r \neq r^{\prime}, r^{\prime \prime}$ by the locality condition. However, from Eq. (3) one sees that

$$
K=\left[\phi_{a}(\mathbf{r}), f_{b c}\right] i \delta^{3}\left(\mathbf{r}^{\prime}-\mathbf{r}^{\prime \prime}\right) .
$$

Choosing $\mathbf{r}^{\prime}=\mathbf{r}^{\prime \prime}=\mathrm{a}$ and $\mathbf{r} \neq a$, one sees that $f_{b c}$ commutes with $\phi_{a}(\mathbf{r})$, except perhaps at $\mathbf{r}=a$. However, repeating the argument with $r^{\prime}=r^{\prime \prime}=b$, $\mathbf{r} \neq \mathbf{b}$ and $\mathbf{b} \neq \mathbf{a}$, implies that $f_{b c}$ commutes with $\phi_{a}(r)$ at $\mathbf{r}=a$ also, i.e., throughout all three-space. Since $\phi_{a}(r)$ represents all the independent variables of the theory, this implies that $f_{b c}$ is a $c$ number. ${ }^{7}$

At this stage, then, $f_{a b}$ is a matrix in $(a b)$ space with $c$-number coefficients. To show that these coefficients are indeed the conventional ones, we compare the Lagrange and Heisenberg field equations, as discussed in Sec. I. Consider the commutator $\left[H[\phi], G_{\phi}\right]$ between the Hamiltonian $H=\int \mathcal{H}(\phi) d^{3} r$ (which is again only a function of the $\phi_{a}$ ) and the Hermitian operator $G_{\phi}=\int \phi_{a} C_{a b} \bar{\delta} \phi_{b} d^{3} r$. Here $C_{a b}$ is any nonsingular (anti) symmetric matrix and

- The derivation that $f$ is a $c$ number does not actually depend on the presence of $\delta^{3}\left(\mathbf{r}-\mathbf{r}^{\prime}\right)$ on the r.h.s. of Eq. (3), but only on the vanishing of the commutator when $r \not r^{\prime}$. However, unless one has the $\delta^{3}$ function, the Lagrange and Heisenberg equations will not be identical.

o S. Schweber, Phys. Rev. 78, 613 (1950).

7 Our definition of completeness of the set of field operators is different from one that has been used in the axiomatic approach [cf. A. L. Licht and J. S. Toll, Nuovo Cimento 21, $346(1961)$ ]. There, a set of fields is called complete if any operator commuting with them at all space-time points is a $c$ number. In text, a set is complete if an operator commuting with the set on a spacelike surface is a $c$ number. From the point of view of formal local field theory, such complete sets should exist (if the theory exists). They represent just the minimal Cauchy data required by the Lagrange equations to uniquely specify the future motion of the system. $\bar{\delta} \phi_{b}$ is an arbitrary infinitesimal quantity (anti) commuting with the $\phi_{a}$ themselves. The operator $G_{\phi}$ may be viewed as the generator of a unitary transformation $U \simeq 1+i G_{\phi}$ which sends $\phi_{a}$ into $\phi_{a}+i / 2(f C)_{a b} \bar{\delta} \phi_{b}$. That is,

$$
U^{-1} \phi_{a} U \simeq \phi_{a}-i\left[G_{\phi}, \phi_{a}\right]=\phi_{a}+(f C)_{a b} \bar{\delta} \phi_{b},
$$

upon using Eq. (3) to evaluate the commutator. Therefore,

$$
U^{-1} H[\phi] U \simeq H[\phi+(f C) \bar{\delta} \phi] .
$$

To first order, then, Eq. (7) reads

$$
\begin{aligned}
-i\left[G_{\phi}, H\right]=H[\phi+f C \bar{\delta} \phi]-H[\phi] & \\
& =\int d^{3} r(\delta H / \delta \phi) f C \bar{\delta} \phi .
\end{aligned}
$$

On the other hand, using the fact that $H$ generates the time motion, we have ${ }^{8}$

$-i\left[G_{\phi}, H\right]=-i \int[\phi, H] C \tilde{\delta} \phi=\int d^{3} r \dot{\phi} C \dot{\delta} \phi$.

Since the Lagrange equation reads, in first-order form, ${ }^{9}$

$$
\dot{\phi}=(\delta H / \delta \phi) A^{\circ},
$$

where $A^{0}$ is a particular nonsingular matrix characteristic of the spin of the system, Eqs. (8) and (10) together imply that $f=A^{0}$ (since $C$ has an inverse). This value of $f$ is, in fact, the conventional one. For scalar theory, for example,

$$
\begin{gathered}
\phi_{a}=(\phi, \pi) ; \\
A^{0}=\left(\begin{array}{rr}
0 & -1 \\
1 & 0
\end{array}\right),
\end{gathered}
$$

so that

$$
\begin{aligned}
& {\left[\phi(\mathbf{r}), \phi\left(\mathbf{r}^{\prime}\right)\right]=0=\left[\pi(\mathbf{r}), \pi\left(\mathbf{r}^{\prime}\right)\right],} \\
& {\left[\phi(\mathbf{r}), \pi\left(\mathbf{r}^{\prime}\right)\right]=i \delta^{3}\left(\mathbf{r}-\mathbf{r}^{\prime}\right) .}
\end{aligned}
$$

In particle systems, the above type of derivation does not determine $f$ uniquely, since the locality and translational invariance conditions are not, in general, available. Thus in field theory these conditions imply that the $f_{a b}$ for two different "degrees of freedom," represented by the fields at two different

\footnotetext{
${ }^{8}$ Note (as shown in the Appendix of reference 1) that $\bar{\delta} \phi_{a}$ must commute with $H$ even if it anticommutes with some of the $\phi_{b}$ (which is the case for Fermi fields). Briefly, $\bar{\delta} \phi_{a}$ would fail to commute with an odd power of Fermi fields in $H$, but if such terms existed they would also give rise to nonlocal parts in the Heisenberg equations of motion, in contradiction with the locality assumption.

${ }^{9}$ For the independent field variables $\phi_{a}$, the Lagrange equations in first-order form take on the simple Hamiltonian structure of Eq. (10) (see reference 1), where the variational derivative in Eq. (10) is identical to the one defined in Eq. (8).
} 
space points, is the same (i.e., $f_{a b}$ is independent of $r$ ). In ordinary quantum mechanics, no simple requirement forcing $f$ to be the same for all the (discrete) degrees of freedom is available. ${ }^{10}$ Of course, to the

${ }^{10}$ Naturally, if one postulates $f$ to be the same for each degree of freedom in the particle case, the canonical results then follow, except in the one-dimensional case (e.g., Wigner's example). The latter system is anomalous since the derivation in text clearly requires more than two independent operators in the complete set [cf. Eq. (4)]. extent that ordinary quantum mechanies can be viewed as the limit of local field theory, our results also apply to it.

Finally, it should be noted that the derivation used here did not utilize the spin-statistics connection. From the result $f=A^{0}$, the connection then follows as a consequence of the symmetry properties of $A^{0}$ and certain assumptions on TCP invariance, as in reference 1 .

\title{
High-Energy Behavior of a Certain Class of Scattering Amplitudes in Perturbation Theory
}

\author{
N. H. Fuchs* \\ Department of Physics and Laboratory for Nuclear Science, Massachusetts Institute of Technology, \\ Cambridge, Massachusetts \\ (Received 7 January 1963)
}

The high-energy behavior for a certain class of Feynman diagrams is determined. The results obtained indicate that Regge-type behavior of scattering amplitude may be associated with the existence of $M$-particle bound states for arbitrary $M$.

\section{INTRODUCTION}

T T has recently been proposed by Gell-Mann and Goldberger ${ }^{1}$ that conventional field theory may predict high-energy behavior of scattering amplitudes in agreement with the existence of Regge poles. $^{2}$ The work of Lee and Sawyer ${ }^{3}$ on the BetheSalpeter equation in the ladder approximation, and that of Federbush and Grisaru, and Polkinghorne ${ }^{4}$ on high-energy behavior in perturbation theory lends credence to this proposal.

In the present work we investigate the highenergy behavior of a class of scattering amplitudes using methods of field theory developed by FG. The relationship between our results in perturbation theory and the concepts of Regge polology is discussed.

* National Science Foundation Predoctoral Fellow.

1 M. Gell-Mann and M. L. Goldberger, Phys. Rev. Letters 9, 275 (1962)

${ }^{2}$ A. Bottino, A. M. Longoni, and T. Regge, Nuovo Cimento 23, 954 (1962)

${ }_{3}^{3}$ B. W. Lee and R. E. Sawyer, Phys. Rev, 127, 2266(1962). (Hereafter denoted by LS)

${ }^{4}$ P. G. Federbush and M. T. Grisaru, to be published. (Hereafter denoted by FG); J. C. Polkinghorne, J. Math. Phys. 4, 503 (1963).

\section{ASYMPTOTIC BEHAVIOR}

We consider the diagram of Fig. 1 and calculate the behavior of the corresponding amplitude for large $s$ and fixed $t$, where we define:

$$
\begin{gathered}
s=\left(K_{M 0}-K_{M, N+1}\right)^{2}, \quad t=\left(K_{10}+K_{M 0}\right)^{2}, \\
p q=\mathbf{p q}-p_{0} q_{0} .
\end{gathered}
$$

We take all masses equal to $m$, and the coupling in the Lagrangian is of the form $g_{(3)} \phi^{3}+g_{(4)} \phi^{4}$.

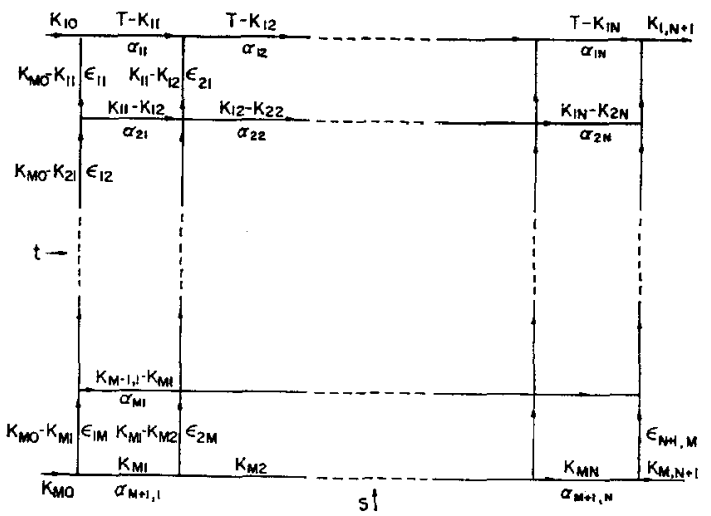

FIG. 1. $T=K_{10}+K_{M O}=K_{M, N+1}+K_{1, N+1}$. 